\title{
The Assessment Perspectives of Residual Resource and Expansion of the Exploitation Activity Marine Platforms
}

Hasanov RA*
Azerbaijan State University of Oil and Industry, Azerbaijan

*Corresponding author: Azerbaijan State University of Oil and Industry, Azerbaijan, Tel: (+99450) 257-24-01; Email: ramizhasanov52@hotmail.com

\author{
Editorial \\ Volume 2 Issue 6
}

Received Date: August 15, 2018

Published Date: August 27, 2018

\section{Editorial}

Recently, the demand for energy carriers on world markets is growing rapidly. The bulk of the recoverable reserves falls to the share of offshore fields. All this puts the task of ensuring high performance and reliability of marine hydraulic structures and their assets. In this direction, a large information base has been accumulated on research conducted by scientists from different countries. Objectives of this information are analyse of the marines platform residual life operational activity evaluation problem and introduction of the methodology for both assessment and maintenance of the platform required structural integrity for the period of expansion of its operational activity.

According to the data submitted by international operating companies the marine platforms regardless of its designed and installed date on the field with i assets planned for a 25-year service life. This indicates that even within the term of the either contract provides for the need to ensure its working capacity for a long time (about several years) beyond the established quota - 25 years. If we take into account the project on modernization (Production Optimization Project), which provides expansion of operational activity and operation of the foundation and its assets for the period up to 50 years, the operation period beyond the established platform quota may reach about $15-20$ years respectively. At the same time, it should be noted that according to the analysis of fatigue phenomena by the services companies (for example Bomel company [1]) for the foundation of any marine platform -50 years of fatigue strength is established, and the actual operational duration for unknown reasons and without justifications is set by operational companies at the level - 25 years (great paradox??).

In this connection, the task is put on impotence to carry out studies on the revision and reassessment of factors provoking a decrease both of the existing and projected operational potentials, i.e. consumption quality (consumption quality factor- is new phenomena!!) of the marine platforms generally. Hence, for the solution of the issue of extending life of the marine platforms primarily the residual resource and existing constructive-technical resources and its acceptability for both mentioned resources of the intended operational periods and technological expansion capabilities on a database of factors of different nature should be assessed. Therefore, it is necessary to develop and introduce an inspection program for risk assessment of all platform's assets to develop appropriate recommendations and achieve the goal of determining the structural readiness and the possibilities for achieving the highest level of modification.

It is necessary to assess the reliability of structural elements according the data for the period of operation subjected to factors of various nature (technical, technological, seismic, meteorological, etc.) in the course of operation during this period of time and, consequently, maintaining and improving the consumer quality of the platform with its assets for a period the over limit of the operational life, taking into account the requirements of the modernization projects. This means that the functionality of the platform in the conditions and the required time interval should ensure the implementation 


\section{Petroleum \& Petrochemical Engineering Journal}

of its technical parameters without deviating in structural integrity and compliance with the conditions of safety, requirements for environment protection and economic efficiency of operation. Information on reducing the marine platform's consumer quality and the reasons for this reduction will allow to develop and implement a plan and appropriate arrangements for monitoring and inspecting both the surface and marine assets of the platform and draw up a road map of potential risks and subsequently develop regulations to maintain the required level platform's consumer quality. Factors that limit the consumption quality of the platform are geological, meteorological, mechanical, hydrodynamic, etc. nature with the corresponding distribution spectra, causing "aging" under the influence of depressive weather and seismic conditions, manufacturing and installation defects, corrosion mechanisms, local destruction, erosion, poor coverage in flood zones and fatigue failure.

According to existing practice, assesment platform's consumer quality on the whole is recommended to be carried out in two stages: basic (being more pessimistic in ensuring the overall integrity of the marine platforms and leading for risk defining associated with the both platform extension life and expansion operational activity) and additional (being less pessimistic and being considered as a stage of full definition and understanding of the structure and platforms status). It is confirmed that most of the connections in the lower part of the platform have fatigue strength significantly greater than its threshold value (equal not accidentally assumed to 50 years), whereas compounds of the upper base level are more sensitive to fatigue failure and have a fatigue strength less than the threshold value, $<50$ years (the information provided shows that the spread is quite large and has nothing in common with the 25 -year lifetime established by operational companeis!!).

In addition, it is great importance and should be considered that the issue of leaving assets at the production site after suspension of the production phase for stopping and abondonment wells for decommissioning. Traditionally, it depends on the schedules and the method of decommissioning the wells according to Plugging and Abandonment plan -covers from 1 to 8 years (Where and how does this rate and time fit into the operational life of the platform? it isn't taken into account !!). The issues of resources of operational activity, evaluation, maintenance and extending of these resources, as well as the development and implementation of the SIM (structural integrity management) cycle in the initial design and in the case of expansion of technological capabilities for any hydro technical structure like and including the marine platforms is very important for the entire period development of the offshore field [2].

These activities should preferably be carried out by independent experts on a the basis of unified methodological approach that combines the evaluation phases, both project stages and operational cycles, the inspected objects (foundation, topsides, facilities), taking into account the design of the platform and factors determining its structural reliability and residual service life. All these aspects should be taken into account, which will allow to solve the problem of structural integrity at the early stages of design, which can be changed due to aging under the influence of technogenic and natural factors, and create a basis for weighting characteristics for use in realization of the projects for extension service life and extending technological capabilities of the platform. It should be noted that the assessment of the factors influencing and determining the load capacity of the platform makes it possible to determine their ranks and significance in drawing up a plan and an inspection program. The main condition for obtaining the relevant assessments is the complete compliance of the critical security elements (CSE) of the structure with all the requirements of the code for its constructive execution and the standards of the structure indicators (SSI - It is necessary to determine with the list of these indicators !!).

\section{References}

1. Bomel (2007) Chirag-1 Spectral Fatigue Assessment.

2. BS EN ISO 19902:2007, Petroleum and natural gas Industries-Fixed steel offshore structures, pp: 622.

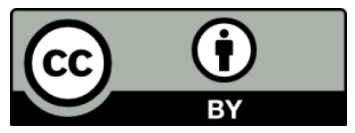

